\title{
A Síndrome de Takotsubo (Síndrome do coração partido): Aspectos hormonais
}

\author{
The Takotsubo Syndrome (Syndrome of the broken heart): Hormonal aspects \\ El Síndrome de Takotsubo (Síndrome del corazón roto): Aspectos hormonales
}

Recebido: 30/01/2021 | Revisado: 05/02/2021 |Aceito: 15/02/2021 | Publicado: 23/02/2021

\author{
Antonio Emanoel Farias Lima \\ ORCID: https://orcid.org/0000-0002-2117-2448 \\ Centro Universitário Santo Agostinho, Brasil \\ E-mail: emanoellima267@gmail.com \\ Francisco Adalberto do Nascimento Paz \\ ORCID: https://orcid.org/0000-0001-6697-1705 \\ Centro Universitário Santo Agostinho, Brasil \\ E-mail: pazadalberto19@hotmail.com
}

\begin{abstract}
Resumo
A síndrome de Takotsubo é tida como uma insuficiência cardíaca aguda reversível que é resultante do atordoamento miocárdico catecolaminérgico, tendo como principal sintomatologia a dor no peito e dispneia. O estudo objetivou buscar na literatura evidências sobre a influência hormonal no desencadeamento da síndrome de Takotsubo. Foi realizada uma revisão integrativa da literatura com busca na Biblioteca Virtual em Saúde (BVS), usando os descritores Cardiomiopatia de Takotsubo, Catecolaminas, diagnóstico; sendo inclusos artigos completos, disponíveis em português, inglês ou espanhol, publicados entre 2010 e 2020. Após análise criteriosa dos resultados obtidos, foram selecionados 10 estudos para compor a pesquisa. Os resultados mostraram uma forte relação entre a liberação hormonal e o desencadeamento da síndrome. As catecolaminas liberadas a partir de uma hiperestimulação do sistema hipotálamo-hipófise-adrenal exercem efeitos importantes na velocidade da contratilidade e força de contração do músculo cardíaco, resultando em perturbação da função cardíaca e aparecimento da síndrome. Conclui-se ainda que a liberação aumentada desses hormônios é estimulada principalmente por situações de estresse, como trauma emocional e estresse cirúrgico. Destaca-se a necessidade de mais estudos na área, como ensaios clínicos, que forneçam mais evidências dessa atuação hormonal no aparecimento da síndrome de Takotsubo, bem como a realização de estudos nacionais, tendo em vista que a grande maioria dos estudos são internacionais.
\end{abstract}

Palavras-chave: Cardiomiopatia de Takotsub; Catecolaminas; Diagnóstico.

\begin{abstract}
Takotsubo's syndrome is seen as a reversible acute heart failure that results from catecholaminergic myocardial stunning, with chest pain and dyspnoea as its main symptoms. The study aimed to search the literature for evidence about the hormonal influence on the onset of takotsubo syndrome. An integrative literature review was carried out by searching the Virtual Health Library (VHL), using the descriptors Cardiomyopathy of takotsubo, Catecholamines, Diagnosis, including complete articles, available in Portuguese, English or Spanish, published between 2010 and 2020. After careful analysis of the results obtained, 10 studies were selected to compose the research. The results showed a strong relationship between hormonal release and the onset of the syndrome. Catecholamines released from hypostimulation of the hipothalamus-pituitary-adrenal system have important effects on the speed of contractility and the force of contraction of the heart muscle, resulting in disruption of cardiac function and the appearance of the syndrome. It is also concluded that the increased release of these hormones is mainly stimulated by stressful situations, such as emotional trauma and surgical stress. The need for further studies in the area, such as clinical trials, to provide more evidence of this hormonal activity in the onset of takotsubo syndrome, as well as the performance of national studies, considering that the vast majority of studies are international.
\end{abstract}

Keywords: Takotsubo cardiomyopath; Catecholamines; Diagnosis.

\section{Resumen}

El síndrome de Takotsubo se considera una insuficiencia cardíaca aguda reversible que resulta del aturdimiento miocárdico catecolaminérgico, con dolor torácico y disnea como síntomas principales. El estudio tuvo como objetivo buscar en la literatura evidencia sobre la influencia hormonal en la aparición del síndrome de takotsubo. Se realizó una revisión integradora de la literatura mediante la búsqueda en la Biblioteca Virtual en Salud (BVS), utilizando los descriptores Cardiomiopatía de takotsubo, Catecolaminas, diagnóstico, incluyendo artículos completos, disponibles en portugués, inglés o español, publicados entre 2010 y 2020. Después del análisis atentos a los resultados obtenidos, se seleccionaron 10 estudios para componer la investigación. Los resultados mostraron una fuerte relación entre la liberación hormonal y la aparición del síndrome. Las catecolaminas liberadas por la hipoestimulación del sistema 
ipotálamo-pituitario-adrenal tienen efectos importantes sobre la velocidad de la contractilidad y la fuerza de contracción del músculo cardíaco, lo que a como resultado la alteración de la función cardíaca y la aparición del síndrome. También se concluye que la mayor liberación de estas hormonas es estimulada principalmente por situaciones estresantes, como traumas emocionales y estrés quirúrgico. La necesidad de realizar más estudios en el área, como ensayos clínicos, para aportar más evidencias de esta actividad hormonal en la aparición del síndrome de takotsubo, así como la realización de estudios nacionales, considerando que la gran mayoría de estudios son internacionales.

Palabras clave: Miocardiopatía de Takotsubo; Catecolaminas; Diagnóstico.

\section{Introduçãa}

A primeira descrição da síndrome de Takotsubo tem sido datada de 1990, feita pelo doutor Hikaru Sato, descrevendo uma disfunção ventricular esquerda que se assemelhava à forma de um antigo vaso japonês usado para pescar polvo (tako = polvo, $t s u b o=$ vaso). Durante aproximadamente trinta anos essa doença recebeu diversas nomenclaturas, tais como disfunção apical transitória, discinesia apical transitória, síndrome do balão apical transitório, coração partido ou cardiomiopatia por estresse. Hoje, é tratada como sendo uma síndrome de insuficiência cardíaca aguda reversível que é resultante do atordoamento miocárdico catecolaminérgico, primário, nos casos onde o paciente foi internado devido ao quadro clínico de Takotsubo, ou secundário, nas situações em que foi hospitalizado por outra causa e, em seguida, apresentou a síndrome de Takotsubo (Espinoza-Alva et al., 2019).

A literatura relata como principais características de sua apresentação clínica, a dor no peito, semelhante aos casos de angina e dispneia, sendo importante realizar o diagnóstico diferencial de uma síndrome coronariana aguda clássica. A síndrome de Takotsubo é encontrada predominantemente em mulheres na pós-menopausa, e tem como fator predominante o estresse emocional, na maioria dos casos. Sua etiologia tem apresentado controvérsias entre os estudos, contudo, tem sido apontado que o excesso de catecolamina em circulação é o mecanismo mais aceitável. As variações na apresentação da síndrome têm sido cada vez mais relatadas, com destaque para os casos descritos como apical, ventricular médio, basal e focal ou a síndrome de Takotsubo invertida, que é caracterizada por ápice hiperdinâmico e acinesia da base, apresentação morfologicamente inversa ao que é observado nos casos comuns (Barbosa et al., 2019).

Como mencionado, muitos mecanismos fisiopatológicos têm sido apresentados no intuito de explicar a síndroma de Takotsubo, como origem em consequência de doença aterosclerótica oculta, presença de espasmo de multivasos ou a disfunção microvascular. Porém, como descrito anteriormente, a principal hipótese para explicar a ocorrência da síndrome é resultante do excesso de catecolaminas que, por sobrecarga de cálcio nos miócitos cardíacos, leva a uma perturbação transitória da contração e função ventriculares. Pode haver a participação do stress emocional, destacando a importância que há em que os profissionais de saúde conheçam o contexto biopsicossocial e familiar dos seus pacientes (Morgado et al., 2019).

Dessa forma, tem-se hoje que a disfunção miocárdica induzida pelo aumento da catecolamina é o mecanismo mais provável para a síndrome. A sua forte associação ao estresse elevado tem direcionado os estudos para o sistema nervoso autônomo, já que as evidências são sugestivas de uma resposta reflexa controlada por esse sistema, tendo em vista que os pacientes com Takotsubo apresentam níveis mais elevados de catecolamina do que aqueles com infarto agudo do miocárdio. Assim, a resposta elevada da catecolamina é apontada como fator fundamental para a ocorrência dessa cardiomiopatia, considerando ainda os efeitos orgânicos no miocárdio (Farias et al.,2018).

O diagnóstico da doença é realizado com base em critérios propostos, como a identificação de alterações transitórias na contratilidade ventricular esquerda, com ou sem envolvimento apical, estendendo-se além do território de uma artéria coronária específica. Deve haver ausência de doença coronariana obstrutiva ou evidência angiográfica de ruptura aguda da placa, novas alterações eletrocardiográficas (elevação do segmento ST e / ou inversão da onda T) ou elevação modesta da troponina, bem como ausência de feocromocitoma ou miocardite. O ecocardiograma é fundamental para o diagnóstico da doença, tendo três padrões ecocardiográficos definidos: invertido, medioventricular e apical. Destaca-se que essa síndrome é 
vista como uma doença benigna, com recuperação notável da função sistólica do ventrículo esquerdo ao longo do tempo (Vergara, Lescano \& Rossi, 2018).

Dessa forma, considerando que a síndrome de Takotsubo é uma doença cardíaca que causa danos funcionais ao coração e que deve ser tratada com atenção, torna-se necessário conhecer sua etiologia para identificar as formas de tratar a doença e garantir melhor qualidade de vida aos que são acometidos por essa patologia, de maneira a reduzir a mortalidade e as complicações em consequência das alterações cardíacas ocorridas. Nesse contexto, este estudou objetivou buscar na literatura evidências sobre a influência hormonal no desencadeamento da síndrome de Takotsubo.

\section{Metodologia}

Trata-se de um trabalho de revisão integrativa da literatura de caráter qualitativo. De acordo com Pereira et al. (2018), métodos qualitativos são aqueles que possuem importância na interpretação do pesquisador que apresenta sua opinião acerca do fenômeno do estudo. Segundo Bento (2012), esse método de estudo é um processo sistemático de investigação onde o pesquisador busca informações sobre determinado problema, e sobre os quais realiza a análise e interpretação, que possibilitam adquirir conhecimentos sobre a situação atual do tema em questão, permitindo ainda identificar possíveis problemas, lacunas e formar ideias sobre a temática estudada.

A busca de dados foi realizada nas bases de dados online da Biblioteca Virtual em Saúde, à qual estão indexados os bancos de dados Scientific Electronic Library Online (SciELO), Literatura Latino-americana e do Caribe em Ciências da Saúde (LILACS), e Medical Literature Analysisand Retrieval System Online (MEDLINE). Como descritores da pesquisa foram utilizadas as palavras "Cardiomiopatia de Takotsubo" and "Catecolaminas" and "Diagnóstico", todas encontradas no Decs (Descritores em Ciências da Saúde).

Para a busca dos artigos, foram considerados apenas os artigos que possuam o texto completo, que estejam disponíveis na língua portuguesa, inglesa ou espanhola, publicados nos últimos 10 anos (2010-2020), sendo usados apenas artigos encontrados nas bases de dados citadas neste trabalho. Foram excluídos da pesquisa todos os trabalhos cuja temática fugiam do tema apresentado, os que estavam apenas com resumos disponíveis, que não possuem versão nos idiomas citados, e que foram publicados em período anterior a 2010, repetidos nas bases de dados, e documentos como teses, monografias e dissertações.

$\mathrm{Na}$ análise dos resultados foram considerados todos os critérios de seleção, sendo selecionados para compor o estudo somente artigos científicos que apresentaram informações pertinentes à pesquisa, e que responderam ao objetivo do estudo (Figura 1). A análise ocorreu mediante leitura completa de todos os estudos selecionados na íntegra, e foram caracterizados a partir de informações como ano de publicação, base de dados, periódico, tipo de estudo, objetivos da pesquisa, autores, título do artigo. 
Figura 1 - Apresentação da busca dos estudos nas bases de dados. Teresina - PI, 2021.

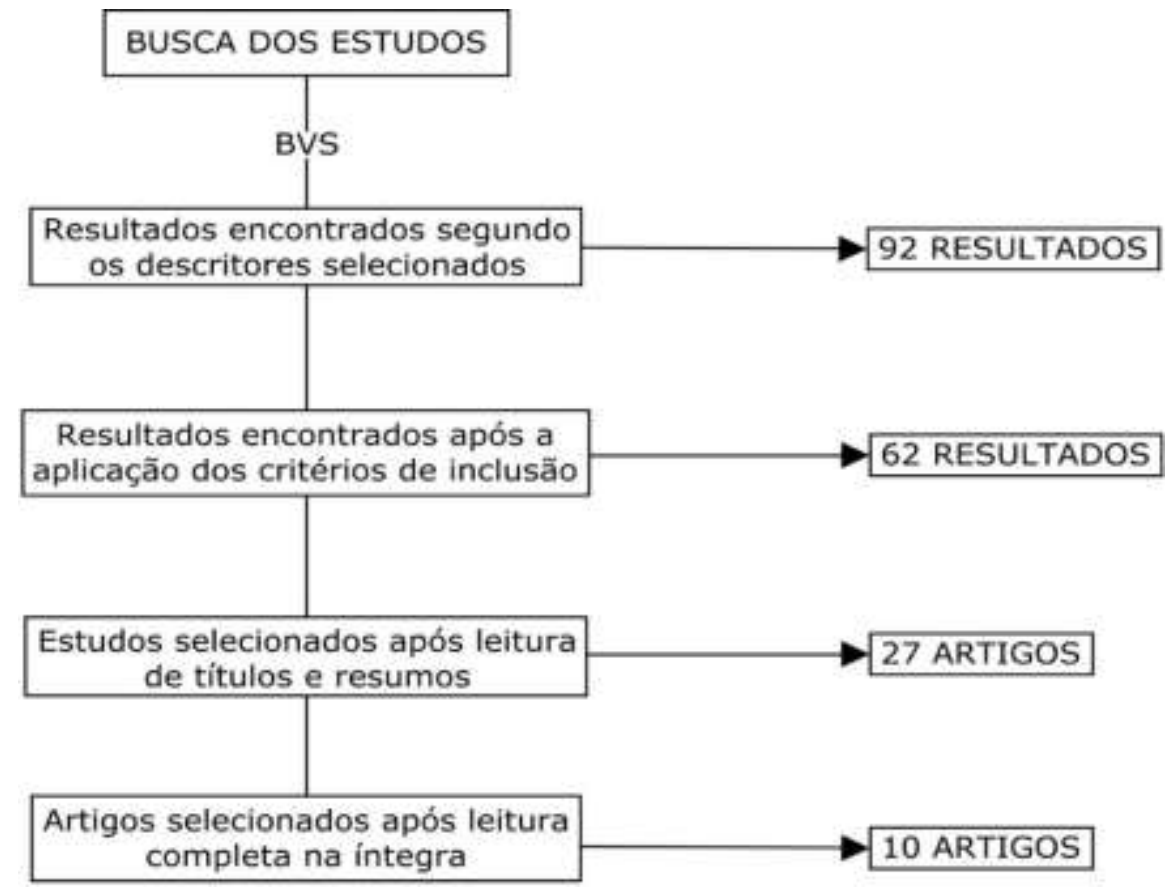

Fonte: BVS.

\section{Resultados e Discussão}

Os estudos selecionados foram organizados em forma de quadro e tabela para apresentação do perfil das publicações. A Tabela 1 apresenta os estudos caracterizados de acordo com o ano de publicação, base de dados e idioma. O ano com maior concentração de estudos foi 2015 , com $30 \%$ das publicações. $90 \%$ dos estudos foram encontrados na Medline via BVS, e $90 \%$ dos estudos estavam em idioma inglês. Esses dados evidenciam o maior contingente de publicações em âmbito internacional.

Tabela 1 - Caracterização dos estudos de acordo com o ano, base de dados e idioma de publicação. Teresina - PI, 2021.

\begin{tabular}{lll}
\hline \multicolumn{1}{c}{ Variáveis } & N & $\%$ \\
Ano de publicação & 2 & 20 \\
2014 & 3 & 30 \\
2016 & 2 & 20 \\
2018 & 1 & 10 \\
2019 & 1 & 10 \\
2020 & 1 & 10 \\
Base de dados & 1 & 10 \\
Lilacs & 9 & 90 \\
Medline & 1 & 10 \\
Idioma & 9 & 90 \\
\hline Português & 9 & \\
Inglês & 9 & 10 \\
\hline
\end{tabular}


O Quadro 1 abaixo apresenta os estudos distribuídos de acordo com o título, periódico e autores da publicação. Em relação ao periódico de publicação, observou-se que a revista com maior número de publicações foi a International journal of cardiology (30\%). A partir dos títulos das pesquisas, foi possível observar que os autores focaram principalmente o efeito das catecolaminas na fisiopatologia da síndrome de Takotsubo e a influência do estresse na sua origem.

Quadro 1 - Apresentação dos estudos selecionados de acordo com o título, periódico e autoria da publicação. Teresina - PI, 2021.

\begin{tabular}{|c|c|c|}
\hline TÍTULO DO ESTUDO & PERIÓDICO & AUTORES \\
\hline $\begin{array}{l}\text { Síndrome de Takotsubo: Uma Doença } \\
\text { Recorrente? / Takotsubo Syndrome: A } \\
\text { Recurrent Disease? }\end{array}$ & Arq. bras. Cardiol & $\begin{array}{l}\text { Fernandes, Fábio; Montera, Marcelo } \\
\text { Westmoreland. }\end{array}$ \\
\hline $\begin{array}{l}\text { A case of catecholamine-induced } \\
\text { cardiomyopathy treated with } \\
\text { extracorporeal membrane oxygenation. }\end{array}$ & BMJ Case Rep & $\begin{array}{l}\text { Garla, Vishnu Vardhan; Gosi, Shiva; Kanduri, } \\
\text { Swetha; Lien, Lillian. }\end{array}$ \\
\hline $\begin{array}{l}\text { Clinical outcomes associated with } \\
\text { catecholamine use in patients diagnosed } \\
\text { with Takotsubo cardiomyopathy. }\end{array}$ & BMC Cardiovasc Disord & $\begin{array}{l}\text { Ansari, Uzair; El-Battrawy, Ibrahim; Fastner, } \\
\text { Christian; Behnes, Michael; Sattler, Katherine; } \\
\text { Huseynov, Aydin; Baumann, Stefan; Tülümen, } \\
\text { Erol; Borggrefe, Martin; Akin, Ibrahim. }\end{array}$ \\
\hline $\begin{array}{l}\text { Takotsubo cardiomyopathy systematic } \\
\text { review: Pathophysiologic process, clinical } \\
\text { presentation and diagnostic approach to } \\
\text { Takotsubo cardiomyopathy. }\end{array}$ & Int J Cardiol & Ono, Ryohei; Falcão, L Menezes. \\
\hline $\begin{array}{l}\text { Improving the understanding of } \\
\text { Takotsubo syndrome: consequences of } \\
\text { diagnosis and treatment. }\end{array}$ & $\begin{array}{l}\text { Expert Rev Cardiovasc } \\
\text { Ther }\end{array}$ & Tanabe, Yasuhiro; Akashi, Yoshihiro J. \\
\hline $\begin{array}{l}\text { Epidemiology and pathophysiology of } \\
\text { Takotsubo syndrome. }\end{array}$ & Nat Rev Cardiol & $\begin{array}{l}\text { Akashi, Yoshihiro J; Nef, Holger M; Lyon, } \\
\text { Alexander R. }\end{array}$ \\
\hline Stress-induced cardiomyopathy. & Crit Care Med & $\begin{array}{l}\text { Boland, Torrey A; Lee, Vivien H; Bleck, } \\
\text { Thomas P. }\end{array}$ \\
\hline $\begin{array}{l}\text { Plasma catecholamine levels in patients } \\
\text { with takotsubo syndrome: Implications } \\
\text { for the pathogenesis of the disease. }\end{array}$ & Int J Cardiol & Y-Hassan, Shams; Henareh, Loghman \\
\hline $\begin{array}{l}\text { Current hypotheses regarding the } \\
\text { pathophysiology behind the takotsubo } \\
\text { syndrome. }\end{array}$ & Int J Cardiol & $\begin{array}{l}\text { Redfors, Björn; Shao, Yangzhen; Ali, Anwar; } \\
\text { Omerovic, Elmir. }\end{array}$ \\
\hline $\begin{array}{l}\text { A single pathophysiological pathway in } \\
\text { Takotsubo cardiomyopathy: } \\
\text { Catecholaminergic stress. }\end{array}$ & Arch Cardiovasc Dis & $\begin{array}{l}\text { Coupez, Elisabeth; Eschalier, Romain; Pereira, } \\
\text { Bruno; Pierrard, Romain; Souteyrand, Géraud; } \\
\text { Clerfond, Guillaume; Citron, Bernard; Lusson, } \\
\text { Jean-René; Mansencal, Nicolas; Motreff, } \\
\text { Pascal. }\end{array}$ \\
\hline
\end{tabular}

Fonte: BVS.

Todos os estudos apontaram para influência das catecolaminas na fisiopatologia da síndrome de Takotsubo, estando fortemente associadas a situações de estresse. Fernandes e Montera (2020), descreveram que a hiperativação do sistema hipotálamo-hipófise-adrenal em resposta a situações de estresse leva ao alto nível de catecolamina, tendo relação importante com a fisiopatologia da síndrome.

Para Garla et al. (2019), a ação das catecolaminas (epinefrina e norepinefrina) sintetizadas na medula adrenal, gânglios simpáticos e cérebro sobre os receptores $\alpha$-adrenérgico e $\beta$-adrenérgico podem ter relação com o desencadeamento da 
síndrome de Takotsubo, já que esses hormônios, quando atuam nestes receptores, medeiam ações como aumento da frequência cardíaca e contratilidade miocárdica.

Ono e Falcão (2016), Tanabe e Akashi (2016), Akashi, Nef e Lyon (2015) e Redfors et al. (2014), também apontaram para a possível relação entre o aumento da ação das catecolaminas e a fisiopatologia da síndrome de takotsubo, podendo ser provocada por condições físicas e médicas que induzem a ativação súbita do sistema nervoso simpático e aumento das catecolaminas, onde as respostas cardiovasculares são causadas pela ativação simpática súbita e aumento nas concentrações de catecolaminas circulantes.

Boland, Lee e Bleck (2015) e Y-Hassan e Henareh (2015) apontaram para a importância do estresse como estímulo para a atividade aumentada das catecolaminas. Dessa forma, relatam que pacientes com síndrome de takotsubo estão associados a níveis elevados de catecolaminas.

No estudo de Ansari et al. (2018) foi observado que pacientes recebendo suporte com catecolaminas apresentaram graus mais elevados de comprometimento circulatório e cardíaco, e o curso da doença foi frequentemente complicado pela ocorrência de diferentes complicações associadas à síndrome, e a mortalidade hospitalar foi significativamente maior no grupo de pacientes recebendo suporte de catecolaminas.

Coupez et al. (2014), observou em sua pesquisa que trauma emocional, estresse cirúrgico, intoxicação adrenérgica e tumor catecolaminérgico foram os principais gatilhos em pacientes com a síndrome de Takotsubo, e destacaram ainda que, independentemente do gatilho, o denominador comum na síndrome foi o estresse catecolaminérgico.

Os resultados dos estudos apontam para o papel das catecolaminas como fator de aparecimento da síndrome de takotsubo. Muitos estudos corroboram com os resultados evidenciados nesta pesquisa, embora ainda não esteja totalmente elucidada a fisiopatologia dessa síndrome.

Assim, embora não se tenha elucidado ainda a etiopatogenia da síndrome, a hipótese mais aceita atualmente tem sido a do aumento das catecolaminas por estimulação simpática, que causaria uma disfunção miocárdica por dano mediado por adrenoceptores, toxicidade direta e disfunção endotelial (Pestana et al., 2019).

Corroborando com o autor acima, Bayon et al. (2019), menciona como hipóteses etiológicas para a síndrome de takotsubo fatores como cardiotoxicidade da descarga do hormônio catecolaminérgico, distúrbio metabólico, circulação microvascular coronariana prejudicada e espasmo da artéria coronária epicárdica. Menciona ainda os sinais e sintomas característicos como dor no peito, biomarcadores elevados de dano miocárdico e alterações eletrocardiográficas que sugerem infarto agudo do miocárdio (elevação do segmento ST e inversão da onda T) com disfunção transitória do ventrículo esquerdo na ausência de estenose significativa ou ruptura de placa na artéria coronária árvore.

$\mathrm{O}$ estresse emocional e físico são fatores tidos como desencadeantes da cardiomiopatia por estresse. Os estressores emocionais mais comuns relatados incluem a morte de um ente querido, agressão e violência, desastres naturais, grandes perdas financeiras, sendo que a maioria envolve um sentimento de destruição, perigo e/ou desespero. Episódios de cardiomiopatia por estresse também podem seguir eventos agradáveis inesperados, "síndrome do coração feliz". Já os estressores físicos relatados incluem doença crítica aguda, cirurgia, dor intensa, sepse e exacerbações de doença pulmonar obstrutiva crônica ou asma. Além disso, distúrbios do sistema nervoso central, como convulsões, acidente vascular cerebral isquêmico ou hemorrágico, encefalite/meningite, traumatismo craniano, síndrome da encefalopatia reversível posterior e esclerose lateral aguda avançada, surgiram como gatilhos (Chazal et al., 2018).

Quanto ao papel do excesso de catecolaminas na patogenia da síndrome de takotsubo, que durante muitos anos tem sido a explicação mais sólida para a ocorrência da doença, tem influenciado no uso preferencial de bloqueadores betas no tratamento. Com mecanismo complexo, inclui reflexos a respostas fisiologias locais e sistêmicas, com estresse agudo e respostas cardiovasculares ao aumento das catecolaminas. Elas possuem um papel central na fisiopatologia da síndrome, 
iniciado geralmente por um estresse repentino, com sinais de ativação simpática presentes na manifestação e que levam a uma ativação simpática extrema. A literatura destaca que dois elementos iniciais da fisiologia devem ser considerados: (1) os centros cognitivos do cérebro, o eixo hipotalâmico da hipófise adrenal (HHP), e a quantidade de adrenalina e noradrenalina que são liberadas em resposta ao estresse; (2) a resposta do sistema cardiovascular (incluindo miocárdio, artérias coronárias e vasculatura periférica), o sistema nervoso simpático à ativação simpática e o aumento repentino de catecolaminas circulantes (Morales-hernández et al., 2016).

Segundo Mimoso (2019), observa em seu estudo que a maioria dos casos da síndrome de takotsubo manifestam-se de forma benigna com um bom prognóstico. Contudo, em alguns casos podem aparecer complicações graves como insuficiência cardíaca, choque cardiogênico, arritmias, regurgitação mitral, formação de trombo, complicações mecânicas e até morte.

Ghadri et al. (2018), escreve que o diagnóstico dessa síndrome é muitas vezes desafiador, pois seu fenótipo clínico pode se assemelhar ao IAM em relação a anormalidades no ECG e biomarcadores. Embora atualmente não exista uma ferramenta não invasiva amplamente estabelecida que permita um diagnóstico rápido e confiável da doença, a angiografia coronariana com ventriculografia esquerda é considerada a ferramenta de diagnóstico padrão-ouro para excluir ou confirmar a síndrome.

Para o tratamento dessa síndrome ainda não há um protocolo padronizado. Atualmente a recomendação é o uso imediato de terapêutica farmacológica preconizada para os casos de infarto agudo do miocárdio (dupla antiagregação plaquetária, nitratos, heparina e betabloqueadores) e, após o diagnóstico da síndrome de takotsubo ser estabelecido, devem ser suspensos os nitratos e antiagregação plaquetária, com a iniciação de tratamento com uso de um inibidor da enzima conversora da angiotensina1. Nos pacientes que apresentarem falência circulatória, não devem ser usados os beta-agonistas ou vasopressores, dando preferência para o uso do suporte circulatório mecânico ou ainda levosimendan (Oliveira et al., 2018).

O prognóstico na síndrome de Takotsubo é excelente, com recuperação quase total em 6 a 8 semanas. Cerca de 1$2 \%$ dos pacientes apresentam recorrência. Taxas de mortalidade de 3-4\% foram relatadas. As complicações ocorrem em pelo menos 1/4 dos pacientes e incluem o seguinte: regurgitação mitral leve a moderada; insuficiência cardíaca esquerda; choque cardiogênico; obstrução da via de saída do ventrículo esquerdo dinâmica; desenvolvimento de trombo mural de VE; arritmias ventriculares; ruptura da parede ventricular e morte (Khalid et al., 2020).

\section{Conclusão}

Com este estudo, viu-se que a síndrome de takotsubo é uma importante patologia que acomete o coração e apresenta etiologias diferentes que devem ser bem conhecidas para implementação adequada do tratamento. Os resultados mostraram uma forte relação entre a liberação hormonal e o desencadeamento da síndrome. As catecolaminas liberadas a partir de uma hiperestimulação do sistema hipotálamo-hipófise-adrenal exercem efeitos importantes ao ligarem-se a receptores cardíacos, tais como aumento de velocidade da contratilidade e força de contração do músculo cardíaco, resultando em perturbação da função cardíaca e aparecimento da síndrome.

Conclui-se ainda que a liberação aumentada desses hormônios é estimulada principalmente por situações de estresse, como trauma emocional e estresse cirúrgico. Contudo, destaca-se a necessidade de mais estudos na área, como ensaios clínicos, que forneçam mais evidências dessa atuação hormonal no aparecimento da síndrome de Takotsubo, bem como a realização de estudos nacionais, tendo em vista que a grande maioria dos estudos são internacionais.

A temática abordada ainda possui poucos estudos e visto que é de grande relevância para comunidade acadêmica, gostaríamos de sugerir que futuros trabalhos sejam aprofundados acerca da Síndrome de Takotsubo, afim de gerar curiosidade e enriquecimento de conhecimento. 


\section{Referências}

Akashi, Y. J., Nef, H. M., \& Lyon, A. R. (2015). Epidemiology and pathophysiology of Takotsubo syndrome. Nature Reviews Cardiology, $12(7), 387$.

Ansari, U., El-Battrawy, I., Fastner, C., Behnes, M., Sattler, K., Huseynov, A., \& Akin, I. (2018). Clinical outcomes associated with catecholamine use in patients diagnosed with Takotsubo cardiomyopathy. BMC cardiovascular disorders, 18(1), 54.

Barbosa, R. R., Silva, M. D., Demian, M. V. D. M., \& Barbosa, L. F. M. (2019). Variant type of stress cardiomyopathy: inverted Takotsubo Syndrome. International Journal of Cardiovascular Sciences, 32(2), 197-200.

Bayon, J., Santás-Älvarez, M., Ocaranza-Sánchez, R., \& González-Juanatey, C. (2019). Assessment with intracoronary pressure and flow guidewire, at baseline and after intracoronary adenosine infusion, in a patient with Takotsubo syndrome. Revista Portuguesa de Cardiologia (English Edition), 38(11), 829. e1.

Bento, A. (2012). Como fazer uma revisão da literatura: Considerações teóricas e práticas. Revista JA (Associação Académica da Universidade da Madeira), 7(65), 42-44

Boland, T. A., Lee, V. H., \& Bleck, T. P. (2015). Stress-induced cardiomyopathy. Read Online: Critical Care Medicine| Society of Critical Care Medicine, 43(3), 686-693.

Campos, M. T. F. D. S., Valente, F. M. Q., Araújo, R. M. A., \& Bressan, J. (2018). Mourning and Takotsubo cardiomyopathy: neu roendocrine implications and nutritional management. Revista da Associação Médica Brasileira, 64(10), 952-959.

Chazal, H. M., Del Buono, M. G., Keyser-Marcus, L., Ma, L., Moeller, F. G., Berrocal, D., \& Abbate, A. (2018). Stress cardiomyopathy diagnosis and treatment: JACC state-of-the-art review. Journal of the American College of Cardiology, 72(16), 1955-1971.

Coupez, E., Eschalier, R., Pereira, B., Pierrard, R., Souteyrand, G., Clerfond, G., \& Motreff, P. (2014). A single pathophysiological pathway in Takotsubo cardiomyopathy: Catecholaminergic stress. Archives of cardiovascular diseases, 107(4), 245-252.

Espinoza-Alva, D., Pampa-Quenta, D. O., Rodríguez-Olivares, R. R., \& Gabino-Gonzáles, G. (2019). Características clínicas y complicaciones del síndrome de Takotsubo en un centro de referencia de la seguridad social peruana. Revista Peruana de Medicina Experimental y Salud Publica, 36, $255-259$.

Faria, S., Cordeiro, C., \& Cavadas, L. F. (2018). Síndroma de Takotsubo em medicina geral e familiar: a propósito de um caso clínico. Revista Portuguesa de Medicina Geral e Familiar, 34(6), 408-412.

Fernandes, F., \& Montera, M. W. (2020). Síndrome de Takotsubo: Uma Doença Recorrente? Arquivos Brasileiros de Cardiologia, 114(3), 484-485.

Garla, V. V., Gosi, S., Kanduri, S., \& Lien, L. (2019). A case of catecholamine-induced cardiomyopathy treated with extracorporeal membrane oxygenation. BMJ Case Reports CP, 12(9), e230196.

Ghadri, J. R., Wittstein, I. S., Prasad, A., Sharkey, S., Dote, K., Akashi, Y. J., \& Templin, C. (2018). International expert consensus document on Takotsubo syndrome (part I): clinical characteristics, diagnostic criteria, and pathophysiology. European heart journal, 39(22), $2032-2046$.

Khalid, N., Ahmad, S. A., Shlofmitz, E., \& Chhabra, L. (2020). Pathophysiology of Takotsubo syndrome. In StatPearls [Internet]. StatPearls Publishing.

Mimoso, J. (2019). Prognosis of Takotsubo syndrome in Portugal. Revista portuguesa de cardiologia: orgao oficial da Sociedade Portuguesa de Cardiologia= Portuguese journal of cardiology: an official journal of the Portuguese Society of Cardiology, 38(5), 359-360.

Morales-Hernández, A. E., Valencia-López, R., Hernández-Salcedo, D. R., \& Domínguez-Estrada, J. M. (2016). Síndrome de Takotsubo. Medicina Interna de México, 32(4), 475-491.

Morgado, G., Gonzalez, F., Oliveira, A. A., \& Fernandes, A. (2019). Uma Outra Causa de Choque Cardiogênico. Arquivos Brasileiros de Cardiologia, 113(6), $1150-1150$

Oliveira, A. M. P., Nascimento, L. S., Nunes, P. B. M., Feil, A. C., Brum, J. C., Peixoto, S. L., \& Junior, N. R. T. (2018). Síndrome de Takotsubo e o uso do InterTAK Diagnostic Score no diagnóstico diferencial com a síndrome coronariana aguda: Relato de um caso. Clinical \& Biomedical Research, 38(4).

Ono, R., \& Falcão, L. M. (2016). Takotsubo cardiomyopathy systematic review: pathophysiologic process, clinical presentation and diagnostic approach to Takotsubo cardiomyopathy. International journal of cardiology, 209, 196-205.

Pereira, A. S., Shitsuka, D. M., Parreira, F. J., Shitsuka, R. (2018). Metodologia da Pesquisa Científica - Literatura em Computação. UFSM.

Pestana, G., Tavares-Silva, M., Sousa, C., Pinto, R., Ribeiro, V., Vasconcelos, M., \& Macedo, F. (2019). Myocardial dysfunction in Takotsubo syndrome: More than meets the eye? Revista Portuguesa de Cardiologia (English Edition), 38(4), 261-266.

Redfors, B., Shao, Y., Ali, A., \& Omerovic, E. (2014). Current hypotheses regarding the pathophysiology behind the takotsubo syndrome. International journal of cardiology, 177(3), 771-779.

Tanabe, Y., \& Akashi, Y. J. (2016). Improving the understanding of Takotsubo syndrome: consequences of diagnosis and treatment. Expert Review of Cardiovascular Therapy, 14(6), 737-748.

Vergara $^{1}$, N., Lescano, A., \& Rossi, A. (2018). Miocardiopatía de Tako-tsubo, una entidad no tan benigna: A propósito de un caso. Insuficiencia cardíaca, 13(4), 192-196.

Shams, Y., \& Henareh, L. (2015). Plasma catecholamine levels in patients with takotsubo syndrome: implications for the pathogenesis of the disease. International journal of cardiology, 181, 35-38. 